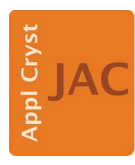

JOURNAL OF

APPLIED

CRYSTALLOGRAPHY

ISSN 1600-5767

Received 24 September 2018

Accepted 21 November 2018

Edited by D. Pandey, Indian Institute of Technology (Banaras Hindu University), Varanasi, India

‡ Deceased

Keywords: $\mathrm{NH}_{4} \mathrm{TiOF}_{3}$; second-order phase transition; crystal and powder X-ray diffraction.

Supporting information: this article has supporting information at journals.iucr.org/j
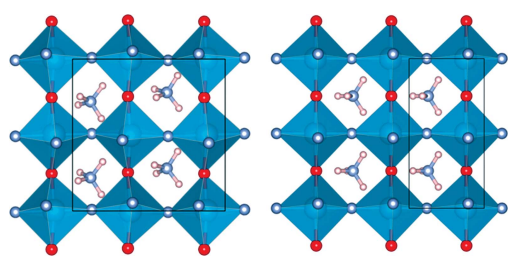

(C) 2019 International Union of Crystallography

\section{Polar and non-polar structures of $\mathrm{NH}_{4} \mathrm{TiOF}_{3}$}

\author{
O. Boytsova, ${ }^{a, b} *$ I. Dovgaliuk, ${ }^{c}$ D. Chernyshov, ${ }^{c, d}$ A. Eliseev, ${ }^{a}$ P. O'Brien, ${ }^{e} \ddagger$ A. J. \\ Sutherland ${ }^{f}$ and A. Bosak ${ }^{g}$
}

a'Department of Chemistry, Lomonosov Moscow State University, Leninskie Gory, Moscow, Russian Federation,
${ }^{\mathbf{b}}$ Kurnakov Institute of General and Inorganic Chemistry of RAS, Leninskii Avenue, Moscow, Russian Federation, ' Swiss-
Norwegian Beam Lines at ESRF, Avenue des Martyrs, Grenoble, France, ${ }^{\mathbf{d}}$ Peter the Great St Petersburg Polytechnic
University, Polytechnicheskaya Street, St Petersburg, Russian Federation, ${ }^{\mathbf{e}}$ Schools of Chemistry and of Materials,
University of Manchester, Oxford Road, Manchester, UK, ${ }^{\mathbf{f}}$ Aston Institute of Materials Research, Aston University,
Birmingham, UK, and ${ }^{\mathbf{g}}$ European Synchrotron Research Facility, Avenue des Martyrs, Grenoble, France. *Correspondence
e-mail: boytsova@gmail.com

Ammonium oxofluorotitanate, $\mathrm{NH}_{4} \mathrm{TiOF}_{3}$, is probably the best known precursor for the synthesis of anatase mesocrystals. Transformation of $\mathrm{NH}_{4} \mathrm{TiOF}_{3}$ into $\mathrm{TiO}_{2}$ through thermal decomposition, accompanied by hydrolysis, preserves some structural features of the precursor. Currently, any discussion of the mechanism of this transformation is difficult, as the exact crystal structure of the starting compound is not available and no intermediate structures are known. This article describes the outcome of single-crystal and powder X-ray diffraction studies, revealing the existence of two polymorphs of the parent $\mathrm{NH}_{4} \mathrm{TiOF}_{3}$ at different temperatures. A second-order phase transition from the polar $P c a 2_{1} \alpha$ phase (1), stable at room temperature, to the Pma $\beta$ phase (2) above $\sim 433 \mathrm{~K}$ has been demonstrated. The direction of the pseudo-fourfold axis in $\mathrm{NH}_{4} \mathrm{TiOF}_{3}$ coincides with the orientation of the fourfold axis of anatase mesocrystals, consistent with a topotactical transformation.

\section{Introduction}

The chemistry of ammonium oxofluorotitanates has been quite widely studied, and structural information has been reported for $\left(\mathrm{NH}_{4}\right)_{3} \mathrm{TiOF}_{5}$ (cubic Fm $3 m$; Massa \& Pausewang, 1978; Udovenko et al., 2003; Udovenko \& Laptash, 2011), $\left(\mathrm{NH}_{4}\right)_{2} \mathrm{TiOF}_{4}$ (monoclinic $P_{1} / \mathrm{m}$; Patarin et al., 1994) and $\mathrm{NH}_{4} \mathrm{TiOF}_{3}$ [orthorhombic Pnma (Laptash et al., 1999; Estruga et al., 2010) or Pbnm (Vasiliev \& Laptash, 2003)]. The topology of structures is rather well known: i.e. isolated octahedra in $\left(\mathrm{NH}_{4}\right)_{3} \mathrm{TiOF}_{5}$, corner-shared chains of octahedra in $\left(\mathrm{NH}_{4}\right)_{2} \mathrm{TiOF}_{4}$ and $2 \mathrm{D}$ layers of corner-shared octahedra in $\mathrm{NH}_{4} \mathrm{TiOF}_{3}$. Amongst these various compounds, $\mathrm{NH}_{4} \mathrm{TiOF}_{3}$ is of special interest as it readily forms anatase mesocrystals through thermohydrolysis and these mesocrystals manifest high and reproducible catalytic activity. However, the details of its structure are not known and there is even an ambiguity in the translational symmetry (Laptash et al., 1999; Estruga et al., 2010; Vasiliev \& Laptash, 2003) - whether doubling along the pseudotetragonal axis takes place.

Typically, the synthesis of $\mathrm{NH}_{4} \mathrm{TiOF}_{3}$ microcrystals, supposedly mesocrystalline, is performed in the presence of a polymer. The methodology initially involved Brij (R) polymer surfactants [i.e. polyoxyethylene (20) cetylether Brij 58; Zhou et al., 2007, 2008; Zhou \& O'Brian, 2008; Boytsova et al., 2018], it was later extended to different polymers [poly(ethylene glycol), PEG; Inoguchi et al., 2012; Boytsova et al., 2017; Liu, Zhang \& Wang, 2013], and finally polymer-free methods were developed (Feng et al., 2010; Ye et al., 2011; Guo et al., 2014; 
Lee et al., 2016; Zhang et al., 2016; Lee \& Lee, 2015; Majima et al., 2016; Fang et al., 2014; Lee \& Shih, 2010; Moriguchi et al., 2001; Jiao et al., 2012). The mechanism of formation - and transformation - is not clear, and one of the reasons is, again, the limited knowledge of the structure.

Here we sought to revisit the initial stage in $\mathrm{NH}_{4} \mathrm{TiOF}_{3}$ transformation, but instead discovered a second-order transition between two noncentrosymmetric phases, not accompanied by chemical transformations. A combination of singlecrystal and powder synchrotron diffraction methods allowed unambiguous determination of space groups and crystal structure solution.

\section{Experiment}

The PEG-assisted synthesis of $\mathrm{NH}_{4} \mathrm{TiOF}_{3}$ was carried out as described previously (Patarin et al., 1994) using PEG 400 or PEG 2000 as growth modifier. Reagents were sourced from the suppliers indicated and were used as received. $\left(\mathrm{NH}_{4}\right)_{2} \mathrm{TiF}_{6}$ (Sigma-Aldrich UK; $0.1 \mathrm{~mol} \mathrm{l}^{-1}$ ), poly(ethylene glycol)s PEG 400 and PEG 2000 (Alfa Chemicals Ltd; $0.8 \mathrm{~mol} \mathrm{l}^{-1}$ ), and $\mathrm{H}_{3} \mathrm{BO}_{3}$ (Alfa Chemicals Ltd; $0.2 \mathrm{~mol} \mathrm{l}^{-1}$ ) were dissolved in distilled water $(30 \mathrm{ml})$ with continuous stirring. After full dissolution of the reagents, the resultant gel was kept at $308 \mathrm{~K}$ for $20 \mathrm{~h}$. A precipitate that formed in this time was isolated by centrifugation/decantation and washed with water $(3 \times 20 \mathrm{ml})$ and acetone $(3 \times 20 \mathrm{ml})$.

The Brij-assisted synthesis of $\mathrm{NH}_{4} \mathrm{TiOF}_{3}$ was carried out by hydrolysis of $\left(\mathrm{NH}_{4}\right)_{2} \mathrm{TiF}_{6}$ on treatment with boric acid in the presence of polyoxyethylene ether following the methodology described above. The molar ratio of the precursors Brij C10 (Sigma-Aldrich UK), $\left(\mathrm{NH}_{4}\right)_{2} \mathrm{TiF}_{6}$ and $\mathrm{H}_{3} \mathrm{BO}_{3}$ in the initial mixture was $2: 4: 6$. The sample obtained with the assistance of Brij C10 is called Brij10 in the current paper.

Both single-crystal and powder data were collected at the ID28 diffraction side station of the European Synchrotron Radiation Facility, with a PILATUS3 X 1M detector, mounted on the rotating arm of an Euler goniostat. In all cases the size of the focal spot was less than $50 \mu \mathrm{m}$; wavelengths of 0.784 , 0.69680 and $0.523 \AA$ were employed in combination with different detector angles to ensure sufficient angular coverage.

For the thermal transformation studies the sample was placed into a $300 \mathrm{~mm}$ quartz capillary (upper end sealed, lower end open to the air) and heated using a hot gas blower calibrated by Ag powder diffraction. For a given temperature 90 frames were collected in shutterless mode with an oscillation of $1^{\circ}$ per image - ranging from room temperature to $\sim 970 \mathrm{~K}$ with $10 \mathrm{~K}$ steps. Images were binned with the $S N B L$ Tool Box (Dyadkin et al., 2016) software and integrated with the Dioptas program (Prescher \& Prakapenka, 2015). Powder data refinement was done using FULLPROF (RodríguezCarvajal, 1993). The refinement of the $\mathrm{NH}_{4}{ }^{+}$rigid bodies was performed using $1.0 \AA \mathrm{N}-\mathrm{H}$ distance constraints. Isotropic atomic displacement parameters for $\mathrm{F}, \mathrm{O}$ and $\mathrm{N}$ atoms were refined independently, and for $\mathrm{H}$ atoms $B_{\text {iso }}$ was increased by 2.0 compared with that of the $\mathrm{N}$ atoms and was refined simultaneously.
Single-crystal data were collected from isolated crystals of typical size $\sim 5 \times 5 \times 1 \mathrm{~mm}$ mounted on a glass fibre (Araldite Rapid epoxy). The data set combines two subsets of 1440 images collected with $0.25^{\circ}$ steps in shutterless mode for two different angles of the detector. CBF files were transformed to ESPERANTO format and treated with the CrysAlis v38.41 software (http://www.rigaku.com/en/products/smc/crysalis). High-resolution reciprocal-space maps were produced by locally developed software of the ID28 beamline.

\section{Results and discussion}

The analysis of powder diffraction data collected below $473 \mathrm{~K}$ for the samples synthesized in the presence of PEG 400, PEG 2000 and Brij C10 shows that neither the diffraction signature of main-phase $\mathrm{NH}_{4} \mathrm{TiOF}_{3}$ nor its thermal behaviour depends on the method of sample preparation (Fig. 1).

Note that (i) no diffraction line position experiences a discontinuous change below $473 \mathrm{~K}$ and (ii) some diffraction lines gradually vanish on heating and gradually return on cooling. These observations should be considered as evidence of a second-order phase transition to a phase with higher symmetry upon heating.

Fig. 2 provides more quantitative evidence on the transition temperature and reversibility of the transition. It tracks the intensity of a peak with a strong temperature dependence normalized with respect to a nearly superposed pair of peaks, which are weakly dependent on temperature.

Thermal cycling (see PEG 2000) shows a gap between the first heating and first cooling, while for the second cycle this gap nearly disappears. A plausible explanation for this observation could be the annealing of defects formed in soft chemical synthesis. The transition temperature can be indicated as $\sim 453 \mathrm{~K}$.

The interpretation of powder diffraction data was greatly facilitated by the single-crystal diffraction data taken for PEG 400 , where the largest high-quality crystals were available $\left(\sim 20 \mathrm{~mm}^{3}\right)$. Analysis of regular extinctions accompanied by twinning (rotation around pseudotetragonal axis) limits the
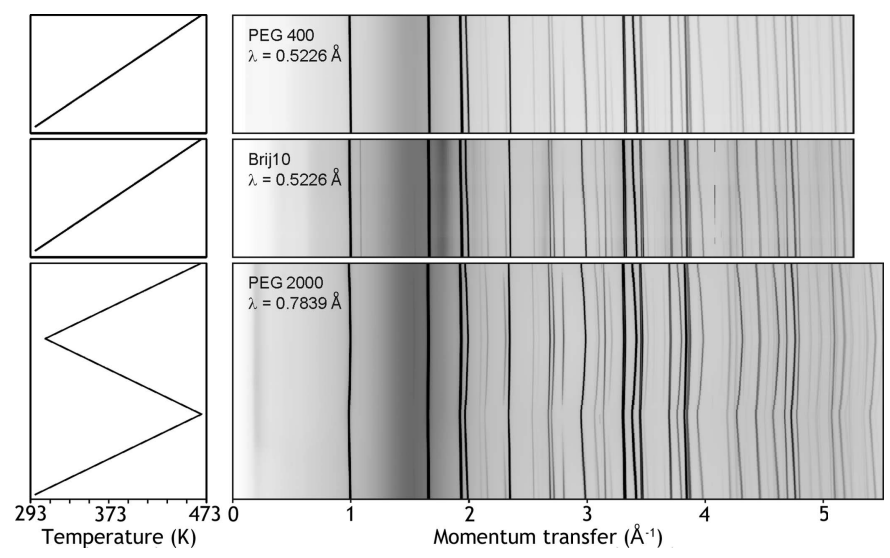

Figure 1

Powder patterns of $\mathrm{NH}_{4} \mathrm{TiOF}_{3}$-based samples traced as a function of temperature. The left panel links the individual patterns to the temperature. 


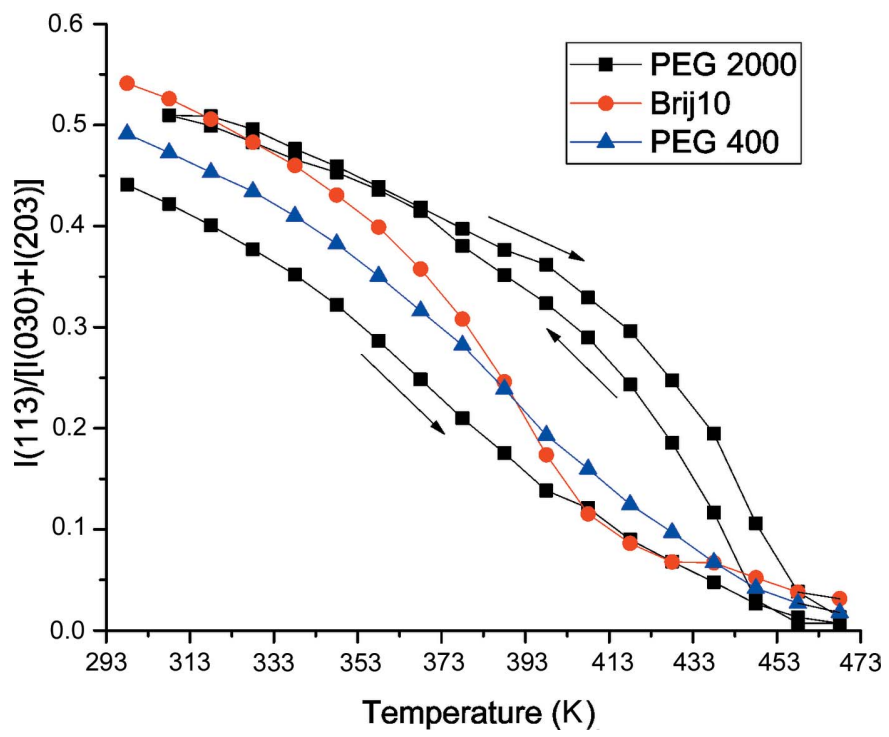

Figure 2

Temperature dependence of the 113 peak normalized to the sum of the 030 and 230 peaks for different samples. Indexing is given as for $P c a 2_{1}$ (see below). The PEG 2000 sample thermal cycling path is indicated by arrows. set of available space groups to $P c a 2_{1}$ and $P b c m$ for the lowtemperature (LT) phase and $P m c 2_{1}, P m a 2$ and $P m m a$ for the high-temperature (HT) phase with half the LT cell size. The two pairs of structures are linked by group-subgroup relationships: $\mathrm{Pca}_{1}(\sim 7.6 \times 6.3 \times 7.6 \AA), \operatorname{Pma} 2(\sim 7.6 \times 6.3 \times$ $3.8 \AA)$ and Pbcm $(\sim 6.3 \times 7.6 \times 7.6 \AA)$, Pmma $(\sim 7.6 \times 3.8 \times$ $6.3 \AA)$. If the piezoresponse reported for the room-temperature phase (Liu, Kumar et al., 2013) is considered, then the first pair must be preferred. No indications of lattice doubling along the pseudotetragonal axis are visible; all the layers of corner-linked octahedra are simply linked by the translation, orthogonal to the layers. Thus the structure does not appear to be similar to the previously reported $\mathrm{NH}_{4} \mathrm{FeF}_{4}$ structure prototype (Laptash et al., 1999; Estruga et al., 2010) and we can disregard earlier discussions about tilt patterns for the adjacent layers (Liu, Kumar et al., 2013).

No reliable identification of $\mathrm{O} / \mathrm{F}$ atoms is available on the basis of the crystallographic data or polar properties of $\mathrm{NH}_{4} \mathrm{TiOF}_{3}$. In the $\mathrm{HT}$ phase, one of the in-plane $\mathrm{Ti}-X$ distances is nearly equal to the apical $\mathrm{Ti}-X$ distance. If we impose the condition that in-plane and apical distances must be the same for a given species, then both these atoms should be fluorine. The absence of apical oxygen is likely to facilitate the thermal hydrolysis process with resultant linking of octahedral layers, i.e. producing the $\mathrm{TiOF}_{2}$ cubic structure known to be one of the decomposition byproducts. Using these assumptions, we performed the refinements of powder diffraction patterns for $\alpha-\mathrm{NH}_{4} \mathrm{TiOF}_{3}$ and $\beta-\mathrm{NH}_{4} \mathrm{TiOF}_{3}$ (see Fig. 3). The latter are in good agreement with the proposed structural models of the octahedral networks and associated ammonia rotations (see Fig. 3). Detailed information on the LT and HT structures derived from powder diffraction refinements is reported in the supplementary CIF.

\section{Conclusions}

We provide the unambiguous determination of the crystal structure of two $\mathrm{NH}_{4} \mathrm{TiOF}_{3}$ polymorphs, subject to a second-order phase transition from $P c a 2_{1}$ to Pma2 on heating at around $\sim 453 \mathrm{~K}$ with the reduction of the formula unit by a factor of 2 . The new structural data provide the basis for attribution of the polar properties of $\mathrm{NH}_{4} \mathrm{TiOF}_{3}$ at room temperature and call for the reinterpretation of some results based on incorrectly assigned translational symmetry. The thermohydrolysis path at higher temperatures, however, cannot be derived from the precursor structure only; it is also

Figure 3

The representation of $\mathrm{NH}_{4} \mathrm{TiOF}_{3}$ crystal structure refinements and corresponding structural models: (a) $\alpha-\mathrm{NH}_{4} \mathrm{TiOF}_{3}$ [space group $P c a 2_{1} ; R_{\mathrm{Br}}=4.7 \% ; a=7.5526$ (1), $b=6.3051$ (1), $c=7.5845$ (1) $\AA$; room temperature, $\lambda=0.69680 \AA ̊$ ] ; (b) $\beta-\mathrm{NH}_{4} \mathrm{TiOF}_{3}$ [space group Pma2; $R_{\mathrm{Br}}=4.0 \% ; a=7.5864$ (2), $b=$ 6.3807 (2), $c=3.80544$ (9) $\AA ; T=498 \mathrm{~K}, \lambda=0.52260 \AA$ ] . Colour code: for $\mathrm{NH}_{4}{ }^{+}$tetrahedra: $\mathrm{N}$ - blue, $\mathrm{H}$ - pink; for $\mathrm{TiOF}_{3}{ }^{-}$octahedra: $\mathrm{O}$ - red, $\mathrm{F}$ - blue. 
necessary to identify the by-products formed in a controlled atmosphere. Since the crystal structure of the starting compound is key for materials properties, careful revision of some hypotheses on the crystallization mechanism, including $\mathrm{TiO}_{2}$ mesocrystals, may be beneficial for improving our knowledge of these materials.

\section{Funding information}

We acknowledge the support of the European Commission (grant PolyCOMP No. 661317) and the RSF (project No. 1873-10212).

\section{References}

Boytsova, O. V., Baranchikov, A. E., Yapryntsev, A. D., Garshev, A. V. \& Ivanov, V. K. (2018). Russ. J. Inorg. Chem. 63, 567-573.

Boytsova, O., Sadovnikov, A. A., Yorov, K. E., Beltiukov, A. N., Baranchikov, A. E., Ivanov, V. K., Zhong, X., Lewis, D. J., O'Brien, P. \& Sutherland, A. J. (2017). CrystEngComm, 19, 3281-3287.

Dyadkin, V., Pattison, P., Dmitriev, V. \& Chernyshov, D. (2016). J. Synchrotron Rad. 23, 825-829.

Estruga, M., Casas-Cabanas, M., Gutiérrez-Tauste, D., Domingo, C. \& Ayllón, J. A. (2010). Mater. Chem. Phys. 124, 904-907.

Fang, Z., Long, L., Hao, S., Song, Y., Qiang, T. \& Geng, B. (2014). CrystEngComm, 16, 2061-2069.

Feng, J., Yin, M., Wang, Z., Yan, S., Wan, L., Li, Z. \& Zou, Z. (2010). CrystEngComm, 12, 3425-3429.

Guo, Y., Li, H., Chen, J., Wu, X. \& Zhou, L. (2014). J. Mater. Chem. A, 2, 19589-19593.
Inoguchi, M., Afzaal, M., Tanaka, N. \& O’Brien, P. (2012). J. Mater. Chem. 22, 25123-25129.

Jiao, W., Wang, L., Liu, G., Lu, G. Q. \& Cheng, H.-M. (2012). ACS Catal. 2, 1854-1859.

Laptash, N. M., Maslennikova, I. G. \& Kaidalova, T. A. (1999). J. Fluor. Chem. 99, 133-137.

Lee, H.-K. \& Lee, S.-W. (2015). Chem. Lett. 44, 604-606.

Lee, H.-K., Okada, T., Fujiwara, T. \& Lee, S.-W. (2016). Mater. Des. 108, 269-276.

Lee, M.-K. \& Shih, T.-H. (2010). J. Phys. D Appl. Phys. 43, 025402.

Liu, Y., Kumar, A., Fan, Z., Zhang, Y., Ke, Q., Zeng, K., Wang, J., Singh, D. J. \& Ong, K. P. (2013). Appl. Phys. Lett. 102, 232903.

Liu, Y., Zhang, Y. \& Wang, J. (2013). CrystEngComm, 15, 791-801.

Massa, W. \& Pausewang, G. (1978). Mater. Res. Bull. 13, 361-368.

Moriguchi, I., Sonoda, K., Matsuo, K., Kagawa, S. \& Teraoka, Y. (2001). Chem. Commun. pp. 1344-1345.

Patarin, J., Marcuccilli-Hoffner, F., Kessler, H. \& Daniels, P. (1994). Eur. J. Solid State Inorg. Chem. 31, 501-511.

Prescher, C. \& Prakapenka, V. B. (2015). High Pressure Res. 35, 223 230.

Rodríguez-Carvajal, J. (1993). Physica B, 192, 55-69.

Udovenko, A. A. \& Laptash, N. M. (2011). Acta Cryst. B67, 447-454.

Udovenko, A. A., Laptash, N. M. \& Maslennikova, I. G. (2003). J. Fluor. Chem. 124, 5-15.

Vasiliev, A. \& Laptash, N. (2003). Institue of Physics, Krasnoyarsk, Russia. ICDD Grant-in-Aid.

Ye, J., Liu, W., Cai, J., Chen, S., Zhao, X., Zhou, H. \& Qi, L. (2011). J. Am. Chem. Soc. 133, 933-940.

Zhang, P., Tachikawa, T., Fujitsuka, M. \& Majima, T. (2016). ChemSusChem. 9, 617-623.

Zhou, L. \& O'Brien, P. (2008). Phys. Status Solidi A, 205, 2317-2323.

Zhou, L., Smyth Boyle, D. \& O'Brien, P. (2007). Chem. Commun. pp. 144-146.

Zhou, L., Smyth-Boyle, D. \& O'Brien, P. (2008). J. Am. Chem. Soc. 130, 1309-1320. 